\title{
La «pierre noire» et la «bulle de savon». Réflexions sur le corps au XXIe siècle
}

\author{
The «Black stone» and the «Soap bubble». \\ Thoughts on the Body in the 21st century
}

\author{
La «piedra negra» y la «pompa de jabón». \\ Reflexiones sobre el cuerpo en el siglo XXI
}

JEAN FRANÇOIS BRAUNSTEIN*

\begin{abstract}
Resumen: Michel Foucault ha transformado nuestra visión del cuerpo. Así como Judith Butler, muchos han querido hallar en él la idea de que "los cuerpos son construidos". La utopía queer o la extraña enfermedad mental de la amputomanía dan cuenta de dicha desmaterialización del cuerpo. Foucault soñaba efectivamente con un cuerpo "pompa de jabón". Esta visión "neo-dualista", muy bien descrita por Ian Hacking, puede llevar hasta un gnosticismo despectivo por la "carne" insignificante que es nuestro cuerpo. Pero para Foucault, en El nacimiento de la clínica, el cuerpo es también una "piedra negra", opaca e impenetrable. Las reflexiones de Canguilhem o la pintura de Bacon nos permiten superar manifiestamente la brutal oposición entre un "cuerpo espiritual", enteramente construido", y un "cuerpo material", simplemente dado.

Palabras clave: Foucault, cuerpo, Canguilhem.
\end{abstract}

\begin{abstract}
Michel Foucault transformed our vision of the body. Many, such as Judith Butler, have been willing to find in Foucault the idea according to which "bodies are constructed". Such a dematerialization of the body finds an echo in the queer utopia or that puzzling mental disorder known as Apotemnophilia. Foucault indeed dreamt of a body conceived as a "soap bubble". That "neo-dualistic" vision, well-described by Ian Hacking, might even result in a Gnosticism that despises the meaningless "meat" our body is made of. But the body also represents for Foucault, in The Birth of the Clinic, a "black stone", opaque and impenetrable. Canguilhem's ideas, or Bacon's paintings, surely allow one to go beyond the crude opposition drawn between a "spiritual body", entirely made up, and a "material body", merely given.
\end{abstract}

Keywords: Foucault, body, Canguilhem.

On a souvent dit que le XXe siècle avait été «le siècle du corps ». C'est le titre d'un très beau recueil de photographies en noir et blanc, de 1900 à 2000, d'Edward Weston à

Fecha de recepción: 29/06/2016. Fecha de aceptación: 02/10/2016.

* Profesor de la Université Paris 1 Panthéon Sorbonne. Sus líneas de investigación son la Filosofía francesa contemporánea y la Historia y Filosofía de las ciencias. Entre sus obras cabe destacar: Canguilhem, histoire des sciences et politique du vivant, Paris, PUF, 2007 y La philosophie de la médecine d'Auguste Comte. Vierge Mère, vaches folles et morts vivants, Paris, PUF, 2009. Jean-Francois.Braunstein@univ-paris1.fr 
Brassai, de Leni Riefenstahl à Cindy Sherman ${ }^{1}$. Mais c'est aussi le point de vue de l'historien Jean-Jacques Courtine dans son introduction au dernier tome, consacré au XXe siècle, de la monumentale Histoire du corps, qu'il a dirigée avec Alain Corbin et Georges Vigarello : « le $\mathrm{XXe}$ siècle a inventé théoriquement le corps », et l'auteur de citer d'abord la psychanalyse, puis l'anthropologie de Marcel Mauss et ensuite la phénoménologie de Merleau-Ponty². Enfin, pour que cette révolution du corps s'accomplisse, il ajoute qu'il fallait deux éléments : d'une part les luttes féministes des années 1970 autour de l'avortement et du slogan : «notre corps nous appartient » et d'autre part « le travail de Michel Foucault» qui a mis en avant « les pouvoirs qui s'exercent sur la chair [...] dans l'horizon historique de la longue durée »3.

L'idée la plus courante serait donc que l'histoire du corps au XXe siècle est celle d'une libération des interdits et des répressions. On parle alors volontiers, à la suite du sociologue Jean Maisonneuve, d'un «corporéisme », largement influencé par Wilhelm Reich : cette « ostentation du corps » comme « objet sensible et sensuel » manifeste depuis les années 1970, s'inscrirait dans une sorte de « Retour de Dionysos » ${ }^{4}$. Il est dès lors curieux d'associer cette perspective à l'oeuvre de Foucault quand on sait combien celui-ci a pu critiquer ce qu'il appela la trop évidente « hypothèse répressive », à laquelle il reconnaît avoir un temps succombé, selon laquelle la sexualité aurait été de plus en plus réprimée par l'ordre capitaliste. Il ironise sur cette prophétie qui fait rapprocher « la révolution et le bonheur ; ou la révolution et un corps autre, plus neuf, plus beau ; ou encore la révolution et le plaisir» ${ }^{5}$.

Certes l'œuvre de Foucault est au cœur de cette révolution du corps que nous avons vécue, et c'est sans doute en tant qu'organisateur d'un séminaire Foucault à la Sorbonne que José Luis Moreno Pestaña m'a fait l'honneur de m'inviter à venir parler devant vous. Mais elle l'est d'une manière beaucoup plus ambivalente qu'il n'y paraît. On a pu à bon droit, dans un livre récent, estimer que l'œuvre de Foucault est une « pensée du corps » ${ }^{6}$. Le terme de corps, au singulier ou au pluriel, est bien présent du début à la fin de l'œuvre foucaldienne, mais les assertions de Foucault sur ce sujet semblent bien souvent mobiles, voire même contradictoires. Une seule chose me semble devoir être sûre, il ne s'agit pas pour Foucault de « libérer le corps » ni même « les corps ».

Sans doute aussi n'a-t-on pas lu la totalité de l'œuvre de Foucault sur cette question avec la même attention. C'est pour souligner ces points de vue apparemment contradictoires de Foucault sur le corps que j'ai choisi pour titre de ma communication deux images, qu'on ne peut guère imaginer plus antithétiques, celle de la «bulle de savon » et celle de la «pierre noire », qui ont toutes deux été employées pour évoquer le corps. Je les ai bien sûr trouvées toutes deux sous la plume de Michel Foucault.

1 Ewing, A., Le siècle du corps, Paris, Editions de La Martinière, 2000.

2 Courtine, J.J., « Introduction » en : Corbin, A., Courtine J.-J., Vigarello, G. (eds.) : Histoire du corps, t. 3, Les mutations du regard. Le XXe siècle, Paris, Editions du Seuil, 2006, p. 7.

3 Ibíd., p. 9.

4 Maisonneuve, J., « Le corps et le corporéisme aujourd'hui », en : Revue française de sociologie, 1976, 17-4, pp. 551-571, p. 551.

5 Foucault, M., La volonté de savoir, Paris, Gallimard, 1976, p. 14.

6 Sforzini A., Michel Foucault. Une pensée du corps, Paris, P.U.F., 2014. 


\section{Mon corps, « topie » impitoyable}

Un des textes les plus curieux dans lequel Foucault évoque la question du corps est une conférence radiophonique datant de 1966, longtemps oubliée, qui a été republiée pour la première fois en 2009 sous le titre : « Le corps utopique ${ }^{7}$. Dans un style qui lui est très inhabituel, avec certaines résonances phénoménologiques, Foucault évoque, en première personne, les sentiments que lui inspire son corps. Un sentiment d'enfermement d'abord : « mon corps, c'est le contraire d'une utopie, ce qui n'est jamais sous un autre ciel, il est le lieu absolu, le petit fragment d'espace avec lequel, au sens strict, je fais corps. Mon corps, topie impitoyable [...]. Et c'est dans cette vilaine coquille de ma tête, dans cette cage que je n'aime pas qu'il va falloir me montrer et me promener ; à travers cette grille qu'il faudra parler, regarder, être regardé ; sous cette peau croupir. Mon corps c'est le lieu sans retour auquel je suis condamné $»^{8}$. Foucault retrouve ainsi tout naturellement la vieille image platonicienne du corps comme prison de l'âme. Un sentiment de dégoût ensuite. Cette prison dans laquelle il est enfermé, ne le révolte même pas, elle lui répugne : « mais tous les matins, même présence, même blessure ; sous mes yeux se dessine l'inévitable image qu'impose le miroir : visage maigre, épaules voûtées, regard myope, plus de cheveux, vraiment pas beau $»$.

Ce sont les tentatives pour sortir de ce corps prison, auquel on ne peut en fait pas échapper, qui sont à l'origine, selon Foucault, de la création des « utopies » de toutes sortes. Le « prestige » de l'utopie tient à cela précisément qu'elle est « un lieu hors de tous les lieux, mais un lieu où j'aurai un corps sans corps, un corps qui sera beau, limpide, transparent, lumineux, véloce, colossal dans sa puissance, infini dans sa durée, délié, invisible, protégé, toujours transfiguré $»^{10}$. C'est cette utopie d'un corps incorporel qui est sans doute « la plus indéracinable dans le cœur des hommes $»^{11}$. Foucault évoque ensuite une autre utopie qui permettrait « d'effacer les corps », celle du « pays des morts », notamment dans la civilisation égyptienne : que sont les momies, ou aussi les masques mycéniens, sinon " l'utopie du corps nié et transfiguré »12 ? Enfin l'utopie «la plus obstinée et la plus puissante » qui peut permettre d'oublier le corps est celle de l'âme, «le grand mythe de l'âme »13. L'utopie de l'âme donne une image de ce que pourrait être un corps enfin « glorieux », un nouveau corps remplaçant ce corps dans lequel nous sommes emprisonnés : « elle est belle mon âme, elle est pure, elle est blanche [...]. Elle durera longtemps mon âme, et plus que longtemps, quand mon vieux corps ira pourrir $»^{14}$. Et Foucault de conclure : «Vive mon âme ! C'est mon corps lumineux, purifié, vertueux, agile, mobile, tiède, frais ; c'est mon corps lisse, châtré, arrondi comme une bulle de savon ${ }^{15}$. Lisse, châtré, arrondi, c'est cela le corps

7 Foucault, M., Le corps utopique suivi de Les hétérotopies, Paris, Lignes, 2009. Une première édition sous forme audio avait été publiée sous le titre Utopies et hétérotopies (Ina-Mémoires vives, Paris, 2004).

8 Ibíd., p. 9-10.

9 Ibíd., p. 10.

10 Ibíd., p. 10.

11 Ibíd..,p. 10.

12 Ibíd., p. 11.

13 Ibíd..,p. 11.

14 Ibíd., p. 12.

15 Ibíd., p. 12. 
idéal. Il est une bulle de savon, ce qui nous rappelle d'ailleurs la vision qui était celle du psychologue Gustav Fechner dans son Anatomie comparée des anges, au début du XIXe siècle : Fechner décrivait les anges comme des corps dépouillés de leurs inégalités et donc ayant « la forme parfaite » d'une « sphère ${ }^{16}$. Ces corps subtils des anges étaient pour lui des « yeux devenus libres ${ }^{17}$, qui se servent de la lumière pour s'exprimer, comme des bulles de savon : " comme la pellicule d'une bulle de savon, la peau de l'ange est, en soi, extrêmement tendre, fine et translucide, et n'est sans doute elle même que le produit d'une condensation [...]. Les anges n'ont donc besoin que de contracter et d'étirer leur peau à leur guise en certains endroits et, de ce fait, ainsi se concentrer ou se diluer, comme la bulle de savon, selon le principe bien connu des physiciens, des couleurs spectrales, pour produire les modulations chromatiques qui sont nécessaires à leur langage»18.

Voilà l'idéal, pour Foucault comme pour Fechner : que nos corps disparaissent et deviennent des bulles de savon ... Il me semble que c'est dans le sens de cette évaporation du corps et de l'oubli de sa matérialité qu'a été bien souvent lu Foucault.

\section{Des corps construits par les pouvoirs}

Cette rêverie autour d'un évanouissement du corps pourrait sembler étrange chez un auteur qui a renouvelé l'histoire de la médecine ou de la psychiatrie en se plongeant dans les archives des corps malades. C'est pourquoi nous aurons aussi à parler, tout à l'heure, d'une autre image, celle de la "pierre noire », que Foucault utilise pour évoquer le corps dans la Naissance de la clinique. Mais il est certain que la conception du corps foucaldien qui a rencontré le plus d'écho dans la culture de la fin du XXe siècle est celle qui est exposée dans les œuvres du milieu des années 1970, notamment dans Surveiller et punir (1975) et la Volonté de savoir (1976). Il s'agit alors d'établir que le corps est façonné par le pouvoir, ou plus exactement que « les » corps sont transformés et disciplinés par «les » pouvoirs : c'est dans Surveiller et punir que Foucault développe l'idée centrale d'une « technologie politique du corps $»^{19}$. Il y aurait un lien direct entre pouvoir et corps : « ce qu'il y a d'essentiel dans tout pouvoir, c'est que son point d'application, c'est toujours, en dernière instance, le corps. Tout pouvoir est physique et il y a entre le corps et le pouvoir politique un branchement direct $»^{20}$. Le pouvoir ne s'applique pas sur la conscience ou la représentation des sujets mais directement sur les corps eux-mêmes : « ce que je cherche, c'est à essayer de montrer comment les rapports de pouvoir peuvent passer matériellement dans l'épaisseur même des corps, sans avoir à être relayés par la représentation des sujets $»^{21}$. Ces « disciplines » s'appliquent au corps d'une manière précise et discrète et non pas « déchaînée »; la « microphysique du pouvoir » repose sur une connaissance finie et détaillée du corps humain.

16 Fechner, G., Anatomie comparée des anges, Paris, Editions de l'éclat, 1997, p. 29.

17 Ibíd., p. 22.

18 Ibíd., p. 34.

19 Foucault, M., Surveiller et punir. Naissance de la prison, Paris, Gallimard, 1975, p. 31.

20 Foucault, M., Le Pouvoir psychiatrique. Cours au Collège de France, 1973-1974, Paris, Gallimard-Seuil, 2003, p. 8 sq., p. 15.

21 Foucault, M., «Les rapports de pouvoir passent à l'intérieur des corps » en : Foucault, M., Dits et écrits, t. III, 1976-1979, Paris, Gallimard, 1994, p. 231. 
La même idée d'une capacité de transformer les corps est au cœur de « l'anatomo-politique du corps humain » présentée dans La volonté de savoir. Alors que la «biopolitique des populations » mettra en place, depuis le milieu du XVIIIe siècle, des «mécanismes régulateurs » des populations, ce pôle du « pouvoir sur la vie », mis en place dès le XVIIe siècle, « a été centré sur le corps comme machine : son dressage, la majoration de ses aptitudes, l'extorsion de ses forces, la croissance parallèle de son utilité et de sa docilité, son intégration à des systèmes de contrôle efficaces et économiques, tout cela a été assuré par des procédures de pouvoir qui caractérisent les disciplines $»^{22}$.

C'est cette idée de « corps dociles », historiquement construits et susceptibles d'être transformés par les pouvoirs qui va surtout être retenue de la leçon de Foucault : les corps ne sont pas donnés, ils sont le résultat de l'histoire, des luttes et des stratégies de pouvoir ou plus largement de la culture. C'est évidemment autour du « genre » et de la lecture de Foucault par Butler que ces emprunts seront les plus évidents et les plus puissamment transformateurs de la société. C'est à La volonté de savoir que Butler se réfère lorsqu'elle souligne que «Foucault nous met en garde contre l'usage de la catégorie de sexe comme une « unité fictive ... (et un) principe causal » $»^{23}$. Et Butler poursuit : « pour Foucault, le corps n'est « sexué » en aucun sens précis du terme avant d'être pris dans un discours qui donne corps à une certaine «idée » de sexe naturel ou essentiel. Le corps ne prend sens dans le discours qu'en situation de pouvoir. La sexualité est une organisation historiquement singulière du pouvoir, du discours, des corps et de l'affectivité. C'est à ce titre que Foucault considère », selon Butler, « que la sexualité produit «le sexe » comme un concept artificiel qui reproduit et dissimule en effet les relations de pouvoir responsables de sa genèse $»^{24}$. Contre Julia Kristeva Butler se sert de Foucault pour dire qu'il faut « revenir de l'illusion d'un corps vrai au delà de la loi »: il n'y a pas de « corps naturel », « le corps construit par la culture sera alors libéré non par un retour vers son passé naturel ou ses plaisirs originels, mais vers un futur ouvert et plein de possibilités $»^{25}$. Butler va très loin en ce sens puisqu'elle estime que les corps sont transformables à volonté. Selon elle, Foucault permet de « démontrer que les catégories fondamentales de sexe, de genre et de désir sont les effets d'une certaine formation de pouvoir ${ }^{26}$ : plus encore, il faut comprendre que c'est le « genre », le sexe ressenti, et les « performances de genre » qui produisent le sexe. Le corps est produit par des discours performatifs.

Cette idée que les corps sont en un sens engendrés par les discours est bien illustrée dans les champs de la «body politics » ou du «body criticism», comme cela apparaît par exemple dans le recueil de textes classique de Margaret Lock et Judith Farqhar, Au-delà du corps propre : le corps n'est plus un donné, « les corps et les vies sont profondément informés par la culture, le discours et la politique ${ }^{27}$, « les corps sont toujours déjà infusés par l'idéologie $»^{28}$, « les corps ne peuvent être réduits à quelque niveau universel organique

22 Foucault, M., La volonté de savoir, Paris, Gallimard, 1976, p. 183.

23 Butler, J., Trouble dans le genre, Paris, La Découverte, 2005, p. 196.

24 Ibíd., 196.

25 Ibíd., 198.

26 Ibíd., p. 53.

27 Ibíd., p. 12.

28 Ibíd., p. 109. 
premier par rapport à la culture et à l'histoire $»^{29}$ sont quelques unes des formules récurrentes des introductions à ces textes. Certains auteurs, pourtant féministes, comme Susan Bordo, qui a travaillé notamment sur «le poids des corps » et les régimes alimentaires, discutent pourtant cette vulgate. Bordo déplore ainsi que le corps soit chez Butler entièrement « textualisé » : elle souligne les « effacements » de la matérialité du corps au profit du nouveau « paradigme de la plasticité $»^{30}$. Selon elle une telle interprétation de Foucault est tout à fait inexacte car il n'existe aucun « antibiologisme » de Foucault. De même, dans un article remarquable, l'historienne du Moyen Age Caroline Bynum cite avec approbation une amie qui s'étonne que dans les études féministes : « le corps se dissolve dans le langage. Le corps qui mange, qui travaille, qui meurt, qui a peur - ce corps n'est simplement pas là $»^{31}$. Je ne reviendrai cependant pas sur les thèses de Butler et sur ces débats qui sont bien connus et qui ne laissent pas indifférent.

Ce qui m'intéresse plutôt, dans la lignée de l'image de la bulle de savon, c'est le caractère de dématérialisation radicale qu'entraîne une telle réflexion, quoique Butler s'en défende. Ainsi elle écrit Bodies That Matter » (traduit en français, Des corps qui comptent) pour réfuter l'idée de ceux dont elle estime qu'ils s'adressent à elle avec condescendance : « si je persistais à croire que les corps étaient en un certain sens construits, peut-être était ce parce que je pensais réellement que les mots avaient à eux seuls le pouvoir de façonner des corps à partir de leur propre substance linguistique ? [...]Si je soutenais que les genres étaient performatifs, cela pouvait signifier que je croyais que chacun, à son réveil, examinait le contenu de son placard ou de quelque espace plus vaste, et y choisissait le genre de son choix, qu'il revêtait ensuite pour la journée avant de le ranger à sa place le soir venu $»^{32}$.

Ce serait ce serait à ce type de critiques $\breve{S}$ à ce type de critiques que Butler aurait voulu répondre dans Bodies That Matter : la question que l'on s'attend à lui voir poser est celle de la «matérialité » du corps. Mais en même temps on ne peut qu'être surpris par la première phrase du livre, très explicite : «j'ai commencé à écrire ce livre en essayant d'examiner la matérialité du corps mais je me suis bientôt aperçu que la pensée de la matérialité me déportait invariablement vers d'autres domaines. Malgré tous mes efforts de discipline je ne parvenais pas à rester sur ce sujet ${ }^{33}$. Et en fait de «matter », de matière, elle reconnaît elle-même qu'elle n'évoque que le second sens, elle traite des corps qui « signifient» et non pas de la «matérialité des corps » qu'elle se refuse à penser.

\section{L'amputomanie ou le corps à la demande}

Pour illustrer cette dématérialisation du corps je souhaiterais évoquer un peu plus en détail, comme une sorte de symptôme, l'oeuvre de l'inventeur du concept de « genre », le

29 Lock, M., Farqhar, J., ed., Beyond the Body Proper, Durham-London, Duke University Press, 2007, p. 2, 12, 109.

30 Bordo, S., Unbearable Weight: Feminism, Western Culture, and the Body, Berkeley-Los Angeles, University of California Press, 2003, p. 246.

31 Bynum, C., «Why All the Fuss about the Body ? A Medievalist's Perspective », Critical Inquiry, 22, 1995, pp. $1-33$, p. 21.

32 Butler, J., Des corps qui comptent, Paris, Editions Amsterdam, 2009, p. 12.

33 Ibíd., p. 11. 
psychologue et sexologue John Money, fondateur de la célèbre Gender Identity Clinic de Johns Hopkins, qui me semble particulièrement symptomatique de ce mouvement vers la dématérialisation. Ses travaux ont depuis été très contestés, aux Etats Unis puis en France, pour d'autres raisons, la fameuse « affaire David Reimer », sur laquelle je ne reviendrai pas. Pourtant des historiennes féministes aussi respectées qu'Anne Fausto-Sterling soulignent l'importance de Money dans l'émergence du genre. Un ouvrage très élogieux a été récemment consacré à Money sous le titre The Man Who Invented Gender ${ }^{34}$. C'est sous sa plume qu'apparaît en effet le terme de genre dans les années 1950 et c'est dans le livre Man and Woman (1972) que John Money et sa collaboratrice Anke Ehrardt «popularisent l'idée que le sexe et le genre sont des catégories séparées : le sexe renvoie à des attributs physiques et est déterminé anatomiquement et physiologiquement. Le genre est vu par eux comme une transformation psychologique du soi, la conviction interne que l'on est soit mâle soit femelle (l'identité de genre) et les expressions comportementales de cette conviction $»^{35}$. Le sociologue français Eric Fassin résume fort bien le sens qu'il convient de donner à cette invention du genre chez Money : «pour John Money, qui participe d'une vision progressiste de la science instituée après la Seconde guerre mondiale en réaction contre les dérives biologiques, c'est bien l'éducation qui fait l'homme ou la femme $»^{36}$. Le sexe est une donnée biologique, le genre est un acquis culturel, indépendant des données du sexe biologique. Les deux ne coïncident pas nécessairement et, dans le cas d'une divergence, c'est l'aspect culturel qui est le plus important. Telle est effectivement, sous une forme très résumée, la thèse de John Money, qui sera reprise et popularisée par le psychanalyste Robert Stoller dans son livre de 1968 Sex and Gender. Le « sexe » réel, objectif, et le "sentiment de genre », subjectif, sont désormais théoriquement déconnectés.

Ce que je voudrais remarquer, et qui n'a à ma connaissance jamais été noté, toujours dans la perspective de la question du corps, c'est que Money est aussi l' "inventeur » d'une nouvelle maladie mentale, qu'il va qualifier d' « apotemnophilie » et qui deviendra plus célèbre sous le nom d'amputomanie. Dans un article fondateur de 1977 Money décrit le trouble très étonnant qu'éprouveraient ceux qui estiment que tel ou tel de leur membre ne leur appartient pas vraiment, qu'il est « en trop », et qui souhaitent donc, pour aller mieux, se faire amputer de ce membre pourtant parfaitement sain ${ }^{37}$. Il pourra s'agir d'un ou de plusieurs doigts, d'un ou de plusieurs bras ou jambes. Il semble à ces malades que cette partie de leur corps leur est étrangère et qu'ils doivent s'en débarrasser pour être enfin euxmêmes. Il n'y avait pas de nom pour une telle affection dans les nomenclatures médicales et Money propose donc de la nommer, d'après des racines grecques, « apotemnophilie », amour (philie) de l'amputation (apotemnein).

34 Goldie, T., The Man Who Invented Gender. Engaging the Ideas of John Money, Vancouver-Toronto, UBC Press, 2015.

35 Fausto-Sterling, A., Sexing the Body. Gender Politics and the Construction of Sexuality, New York, Basic Books, 2000, p. 3.

36 Fassin, E. , «L'empire du genre. L'histoire politique ambiguë d'un outil conceptuel », en : L'Homme, 2008/3 n 187-188, pp. 375-392, p. 375.

37 Money, J., Jobaris, R., Furth, G., « Apotemnophilia: Two Cases of Self-Demand Amputation as a Paraphilia », Journal of Sex Research 13, no. 2 (1977), pp. 115-25. 
Il faut certes être très prudent quant à la prévalence originelle de ce trouble. Dans l'article en question Money se base sur seulement deux cas d'hommes s'étant adressés à lui, dans son service de Johns Hopkins, pour une telle demande d'amputation volontaire d'un membre sain. Le moins que l'on puisse dire est que l'échantillon est réduit, d'autant que l'un de ces patients n'a été connu que "par téléphone et par courrier ». Plus étonnant encore d'un point de vue déontologique, il faut noter que l'un des co-auteurs de cet article avec Money, Gregg Furth, est un psychanalyste qui avoua lui-même qu'il désirait dès son plus jeune âge se faire amputer de la jambe droite. Furth tentera par la suite par deux fois de se faire amputer au Mexique avant de trouver un médecin en Ecosse, Robert Smith, qui veuille bien accepter de l'opérer. Malheureusement pour Furth les autorités sanitaires écossaises interdirent entre temps ce type d'opération.

Tout cela est d'une certaine manière anecdotique et l'on sait que Money est un amateur de « curiosa » médico-sexuelles : ce ne serait que l'un des nombreux cas de «paraphilies » qu'il collectionnait à travers ses ouvrages. Mais le problème est que l'amputomanie, une fois « lancée », a commencé à se répandre de manière quasi épidémique, comme bien d'autres «maladies mentales transitoires ». En ce sens le philosophe Ian Hacking, qui est l'un des rares à s'être intéressé à cette maladie, a pu noter que l'amputomanie est sans doute la première maladie liée à l'Internet. En effet, dans la vie « réelle », il y a fort peu de chances pour que deux personnes estimant urgent de se faire couper une jambe en parfait état se rencontrent et s'échauffent à l'idée de cette amputation. Il n'y a que sur le net qu'il est possible à des personnes souffrant de ce trouble de se reconnaître et d'entrer en relations, voire même à des personnes se sentant «mal dans leur peau » de se dire : je ne vais pas bien, je ne sais pas ce qui m'arrive, mon corps ne me convient pas, mais voilà maintenant je sais, l'apotemnophilie est ma maladie. On rencontrera alors d'autres amputomanes, des médecins prêts à les opérer et des systèmes de sécurité sociale qui s'interrogeront pour savoir si ces opérations doivent être prises en charge par la collectivité. Pour peu que l'on soit déjà versé dans l'expérience des modifications corporelles diverses, comme les tatouages, infibulations ou autres scarifications, la voie sera encore plus facile à suivre et l'amputomanie pourra apparaître comme la suite naturelle de ces expériences de modification corporelle. Il y a là, comme l'a noté Ian Hacking, un « effet de boucle » propre aux sciences humaines : cellesci en créant de nouvelles catégories, par exemple psychiatriques, comme l'amputomanie, « fabriquent des gens » (make up people) qui vont se désigner pour remplir ces cases. Si on dispose d'une catégorie nosologique, de « malades » qui s'organisent pour faire reconnaître ce syndrome, par exemple en la faisant insérer dans le DSM, un chirurgien pour pratiquer l'opération, un organisme de sécurité sociale pour la rembourser, on y est : l'amputomanie existe et l'amputation volontaire d'un membre sain sera un traitement à ce trouble.

Si l'on passe outre le caractère très restreint des observations de Money il faut noter que la ressemblance avec les questions posées par le transsexualisme est assez claire. Le grand antipsychiatre Thomas Szasz en avait eu l'intuition lorsque, s'opposant aux opérations des transsexuels, il s'était posé la question, avant l'épidémie d'amputomanie : « que se passerait-il si un homme allait voir un chirurgien orthopédique, lui disait qu'il se sent comme un droitier emprisonné dans un corps ambidextre et demandait au médecin de lui couper son bras gauche pourtant en parfaite santé ? ${ }^{38}$. Même sentiment de malaise diffus autour de la

38 Szasz, T., « Male and Female Created He Them », New York Times, June 10, 1979. 
question de l'identité, même proposition d'une solution chirurgicale radicale et irréversible, même mise en avant de quelques cas fortement médiatisés qui jouent un rôle d'entraînement. Pour l'instant l'amputomanie a eu moins de succès que le transsexualisme et ont peut espérer que ce succès restera limité.

Mais, au delà de cette démonstration par l'absurde de l'emprise médicale sur nos vies et nos désirs, il y a un autre aspect qui nous intéresse ici. L'amputomanie met en évidence le postulat, le plus souvent inexprimé, qui est au cœur de la plupart des débats sur le genre. L'idée est que seule compte notre conscience d'être ceci ou cela, l'idée que ce membre est en trop pour nous, par rapport à ce que nous estimons devoir être notre « vrai » corps. De même il nous semble que nous sommes ou homme ou femme, ou queer, ou autre, et que c'est cette volonté, cette identité, qui doit déterminer ce qu'est notre corps. Notre désir d'être ceci ou cela est seul juge, le corps n'a qu'à suivre, avec au besoin l'aide de la médecine et de la chirurgie. Le succès assez improbable de cette maladie émergente qu'est l'amputomanie est le signe que ce que n'acceptent plus bon nombre de nos contemporains, c'est que notre corps soit tout simplement déjà là, dans sa matérialité, homme ou femme, blond ou brun, grand ou petit, gros ou maigre, sain ou malade, dans sa simple contingence et dans sa finitude. Si je désire être un homme, une femme, un amputé ou quoi que ce soit d'autre, peu importe ce dont témoigne notre corps matériel, avec ses imperfections et son inéluctable contingence.

\section{Néo-dualisme}

On peut en effet soutenir à bon droit que la pensée contemporaine se caractérise par une séparation de plus en plus radicale entre l'âme et le corps. Comme l'a bien vu le philosophe canadien Ian Hacking -qui donna il y a quelques années au Collège de France un cours sur « Le corps et l'âme au début du XXIe siècle »- « notre corps, sous l'effet de toute une série de nouvelles technologies, devient radicalement autre. Nous redevenons cartésiens, dissociant l'âme du corps, qui apparaît presque comme un objet extérieur à nous» ${ }^{39}{ }_{\gg}$. Un certain nombre de pratiques : greffes, mort cérébrale, marché des organes vivants, changement de sexe, amputomanie, médecine génétique, stocks génétiques en Islande, performances artistiques, ou cyborgs nous conduisent à faire de notre corps un objet, ou un ensemble d'objets, dont il nous est possible de disposer à volonté. Les corps sont désormais à notre disposition et nous disposons, selon Hacking, de « corps néo-cartésiens en morceaux $»^{40}$. Pièces de rechange (Spare Parts) est d'ailleurs le titre d'un ouvrage récent de l'anthropologue Lesley Sharp sur les greffes d'organes ${ }^{41}$. Il semble même que l'on aille plus loin que Descartes puisque celui-ci ne méprisait pas le corps auquel il consacrait tout le volume du traité De l'homme. Mais dans les débats contemporains sur le genre, le transsexualisme ou

39 Hacking, I., «Résumé du cours 2003-2004 au Collège de France. Le corps et l'âme au début du XXIe siècle », Site du Collège de France : https://www.college-defrance.fr/media/ianhacking/UPL2883502379452881562_ Hacking2003_2004.pd

40 C'est le titre d'un article de Ian Hacking, « Our Neo-Cartesian Bodies in Parts », Critical Inquiry, Vol. 34, N 1 (Autumn 2007), pp. 78-105.

41 Sharp, L., Strange Harvest. Organ Transplants, Denatured Bodies and the Transformed Self, Berkeley-Los Angeles-London, University of California Press, 2006. 
l'amputomanie on voit à l'œuvre un véritable indifférence pour le corps qui n'a qu'à suivre la volonté : seuls comptent les désirs ou les identités, les personnalités et les consciences.

L'individu se perçoit comme le propriétaire d'un corps radicalement étranger et modifiable, dont il peut jouer à volonté. C'est aussi le point de vue qu'illustre un autre canadien, cinéaste mais aussi ancien étudiant de Marshall Mac Luhan à Toronto, David Cronenberg, fasciné par les modifications corporelles, les cicatrices et les tatouages. Dans ses premiers films, comme Videodrome, les corps sont déformés, éventrés, reconstruits. Cronenberg joue sur les limites entre l'intérieur et l'extérieur des corps qu'il estime indissociables, réversibles : "Il reste toujours étonnant, à mes yeux, que tout le monde puisse se mettre d'accord sur la beauté d'une femme, mais que beaucoup seraient dégoûtés par le même corps en rayons $\mathrm{X}$, ou auraient la nausée s'ils voyaient le même corps durant une opération chirurgicale [...]. Nous n'avons pas une esthétique totale dans la mesure où nous n'assumons pas l'intérieur de nos corps et la compréhension des organes et de leur fonctionnement $»^{42}$. Il faut selon lui s'intéresser à la «nouvelle chair » offerte par la technologie (« longue vie à la nouvelle chair ! » est le mot d'ordre dans Videodrome) et aux nouveaux types de plaisir qu'elle peut procurer, notamment à travers de nouveaux orifices sexuels qu'il est possible de fabriquer. Emblématique de cette recherche est son adaptation étonnante, profondément dérangeante et troublante, de Crash, le chef d'œuvre d'un autre auteur contemporain qui a mis le corps postmoderne au centre de son œuvre, James Graham Ballard. Ce même Cronenberg est bien conscient de la dimension philosophique de son approche puisqu'il reconnaît : « je deviens très cartésien, vous savez. Descartes était obsédé par le schisme entre l'âme et le corps, et comment l'un est lié à l'autre ${ }^{43}$. Pour ces deux auteurs, Cronenberg et Ballard, l'expérience la plus étonnante de notre temps est celle de ces reconstructions de corps tout à la fois lisses, désincarnés mais aussi fortement érotisés par les blessures qu'ils s'infligent dans des accidents soigneusement planifiés.

Au-delà de l'exemple extrême de l'amputomanie et des fictions affolantes de Cronenberg ou Ballard, il existe bien d'autres pratiques corporelles contemporaines de masse qui manifestent cette volonté de donner un sens à un corps qui n'en a pas. On connaît l'engouement pour une chirurgie esthétique extrémiste qui vise à transformer radicalement des corps. On pourrait donner des exemples qui relèvent de la sociologie du corps et je crois savoir que José Luis Moreno Pestaña travaille sur la question des «fat studies », de l'anorexie, si répandue en Occident, qui manifeste une véritable peur du corps et un refus corrélatif de la sexualité.

On ne peut aussi que constater l'engouement actuel pour le tatouage et les modifications corporelles dans une bonne partie de la jeunesse du monde développé, à laquelle les philosophes n'ont pas assez réfléchi, alors que d'autres, sociologues ou écrivains, y ont été plus attentifs. Foucault, dans la conférence citée plus haut, voyait dans le tatouage un des moyens de donner une signification au corps en le faisant entrer « en communication avec des pouvoirs secrets et des forces invisibles »: « le masque, le signe tatoué, le fard déposent sur le corps tout un langage énigmatique, tout un langage chiffré, secret, sacré, qui appelle sur ce même corps la violence du dieu, la puissance sourde du sacré ou la vivacité du désir.

42 Cronenberg, D., Entretiens avec Serge Grunberg, Paris, Les Cahiers du Cinéma, 2000, p. 106.

43 Sammon, P. M., « David Cronenberg. Article and interview», Cinefantastique, $10:$ 4, Spring 1981, 22. 
Le masque, le tatouage, le fard placent le corps dans un autre espace, ils le font entrer dans un lieu qui n'a pas de lieu directement dans le monde, ils font de ce corps un fragment d'espace imaginaire qui va communiquer avec l'univers des divinités ou avec l'univers d'autrui » ${ }^{44}$. Ce sont des opérations «par lesquelles le corps est arraché à son espace propre et projeté dans un autre espace $»^{45}$. Comment mieux dire que le tatouage permet d'échapper à la radicale contingence du corps, à son insignifiance initiale. Mais en 1966, lorsque Foucault y faisait allusion, ce phénomène n'était encore guère répandu, alors qu'il est devenu aujourd'hui massif dans les sociétés développées où justement il n'y a plus de religions pour justifier le corps ou nous faire attendre sa résurrection comme « corps glorieux ». Au delà d'une recherche assez vaine de la singularité, quand on sait le répertoire limité des tatoueurs, il est surtout question de donner du sens à un corps qui sans cela resterait muet, insignifiant, contingent. Notre corps doit dire quelque chose, doit signifier « notre » personnalité et ne pas être un corps quelconque indifférent à notre «identité » personnelle.

\section{Gnosticisme}

Un autre exemple de changement des attitudes à l'égard du corps qui mériterait réflexion est celui de l'engouement extraordinaire et en très rapide augmentation pour la crémation en Occident. Au delà de ses justifications écologiques ou de ses motifs simplement économiques, on peut sans difficulté y discerner une véritable horreur du corps, sous sa forme la plus impure, celle du cadavre et de son pourrissement. Le corps doit désormais disparaître, se volatiliser au moment de la mort et ne plus subsister lorsque la conscience le quitte. Ce n'est pas par hasard que cette pratique de la crémation a toujours été pratiquée dans des sociétés comme les sociétés hindouiste ou bouddhiste qui n'attachent pas une importance essentielle aux enveloppes corporelles. Un écrivain comme Michel Houellebecq est l'un des rares à avoir relevé ce phénomène massif pour le condamner comme « anthropologiquement impie ». Dans la Carte et le territoire le personnage nommé Houellebecq a demandé à « ne pas être incinéré, mais très classiquement enterré. "Je souhaite que les vers dégagent mon squelette », précisait-il, s'autorisant une notation personnelle dans un texte de facture par ailleurs très officielle ; "j'ai toujours entretenu d'excellentes relations avec mon squelette, et je me réjouis qu'il puisse se dégager de son carcan de chair $»^{46}$. Il est approuvé en cela par un autre personnage assistant aux obsèques qui estime qu'il est « en quelque sorte anthropologiquement impie de disperser les cendres d'un être humain dans les prairies, les rivières ou la mer $\gg^{47}$.

Il serait sans doute possible d'aller plus loin et de penser que notre corps n'est désormais plus seulement perçu comme une machine, un outil à notre service, selon un schéma néo-dualiste, mais plutôt comme quelque chose qui nous fait honte. Plus que d'être à notre disposition, il semblerait que notre corps aujourd'hui nous dégoûte. Ce que notre société ne supporte plus c'est que notre corps soit simplement là, et que nous y soyons nécessairement liés. On connaît l'image qu'employait le jeune Aristote, encore très platonicien, pour dési-

44 Foucault, M., Le corps utopique suivi de Les hétérotopies, Paris, Lignes, 2009, p. 15.

45 Ibíd., p. 16.

46 Houellebecq, M., La carte et le territoire, Paris, Flammarion, 2010, p. 317.

47 Ibíd., p. 3. 
gner le rapport de l'âme au corps : leur relation évoquait pour lui le supplice tyrrhénien où un prisonnier était lié à un cadavre. Le corps est pour nous aussi ce cadavre répugnant, avec qui l'on ne supporte plus de devoir vivre. Il s'agit là d'un véritable retour au gnosticisme, à cette interprétation qui voit dans le corps la marque du mal, de la corruption de l'homme.

On retrouve cette idée dans toutes les réflexions autour de tous les trans- ou post-humanismes. C'est ce que notait il y a déjà longtemps Günther Anders, avec ses remarques sur la « honte prométhéenne » que l'homme peut éprouver face aux machines si parfaites qu'il a fabriquées. Face à ces machines, l'homme semble si mal conçu, si fragile. Il a honte de son origine naturelle, contingente et imparfaite. Dans L'obsolescence de l'homme, le personnage qu'observe Anders dans une exposition de machines « a honte d'être devenu plutôt que d'avoir été fabriqué. Il a honte de devoir son existence - à la différence des produits qui, eux, sont irréprochables parce qu'ils ont été calculés dans les moindres détails - au processus aveugle, non calculé et ancestral de la procréation et de la naissance. Son déshonneur tient donc au fait d' «être né», à sa naissance qu'il estime triviale (exactement comme le ferait le biographe d'un fondateur de religion) pour cette seule raison qu'elle est une naissance. Mais s'il a honte du caractère obsolète de son origine, il a bien sûr également honte du résultat imparfait et inévitable de cette origine, en l'occurrence lui-même $»^{48}$.

Ces remarques annoncent bien sûr le le mépris que les prophètes du post-humain éprouvent pour l'imperfection de notre corps lorsqu'ils la comparent à la perfection des puces en silicium de l'ordinateur. Notre corps c'est ce que William Gibson, auteur de Neuromancien et inventeur du cyberpunk, appelle avec mépris le monde de la «viande » : «le corps, c'était de la viande $»^{49}$. Son héros s'efforce de résister à cet appel de la viande lorsqu'il l'entend encore : « la viande, lui disait une partie de lui-même, c'est la viande qui parle, ignore-la $»^{50}$.

Les autres théoriciens du posthumanisme sont eux aussi en ce sens des nouveaux gnostiques : on retrouve le même dégoût pour la «viande » chez Hans Moravec ou Marvin Minsky, qui remplaceraient volontiers le corps par des microprocesseurs. Pour Minsky « une personne, ce n'est pas une tête, des bras et des jambes. C'est trivial. Une personne c'est un très grand multiprocesseur fait de millions de millions de petits composants qui sont agencés comme des milliers de calculateurs [...]. La chose la plus importante à propos de chaque personne ce sont les données, et parmi ces données les programmes qui sont dans le cerveau. Et un jour vous serez capable de rassembler toutes ces données, de les mettre sur un petit disque, et de les stocker pour des milliers d'années, et ensuite, en branchant tout cela à nouveau vous serez vivant dans le quatrième ou le cinquième millénaire $»^{51}$. Voilà l'avenir radieux annoncé, le downloading de la conscience.

A la rigueur, si l'on devait sauver quelque chose de notre corps, cela ne pourrait être que le cerveau, qui ressemble déjà tellement à une machine informatique. Auguste Comte en avait eu le pressentiment lorsqu'il expliquait que notre cerveau est un organe tout à fait

48 Anders, G., L'obsolescence de l'homme, Paris, Editions de l'Encyclopédies des Nuisances - Editions Ivrea, Paris, 2002, p. 38.

49 Gibson, W., Neuromancien, Paris, La Découverte, 1985, p. 9.

50 Ibíd., p. 181.

51 Minsky, M., « How Computer Science Will Change Our Lives », Nara Conference on Artificial Life (1996) en : Hayles, N. K., How We Became Posthuman, Chicago-London, University of Chicago Press, 1999, p. 244-245. 
exceptionnel, «le seul qui ne peut être remplacé », celui qui a la plus grande longévité aussi : notre cerveau pourrait, selon lui, « user deux corps, et peut-être trois ${ }^{52}$. Et Comte de conclure : «le corps est un simple soutien du cerveau », il n'existe il n'existe qu'en fonction de son utilité pour le cerveau, qui est le seul organe vraiment digne de notre Humanité ${ }^{53}$. Le cerveau est d'ailleurs un organe tellement particulier qu'il récupère bon nombre des caractères de l'âme, dont son immortalité : il existe une véritable immortalité cérébrale, ce que Comte nomme «l'immortalité subjective ». Tant qu'il y a des cerveaux vivants qui gardent le souvenir de leurs prédécesseurs, il n'y a pas à proprement parler, de mort. Au XXe siècle cette idée d'une pérennité des cerveaux sera reprise par des savants comme les époux Vogt en Allemagne, qui envisagèrent de créer un véritable culte autour de leur « Panthéon des cerveaux » et qui affirmaient que l'homme devient, et de plus en plus, « ein Hirntier », un animal cérébral ${ }^{54}$. C'en sera alors fini de la misère corporelle.

\section{La « pierre noire » du corps}

Il semble donc qu'une certaine lecture de Foucault est au diapason d'un courant néodualiste, voire gnostique, qui considère le corps comme inessentiel ou défectueux. Mais les réflexions de Foucault sur le corps ne se réduisent pas aux œuvres des années 1970 et aux lectures qui ont pu en être faites. D'ailleurs, même dans ceux de ses textes qui ont été les plus utilisés dans un sens qui tend à faire des corps des effets du pouvoir, Foucault tient à souligner que reconnaître le caractère historique du sexe n'implique pas «nécessairement l'élision du corps, de l'anatomie, du biologique, du fonctionnel $»^{55}$. Foucault, au moins d'un point de vue programmatique, est clair, il ne s'agit pas de faire une « histoire des mentalités » : « le but de la présente recherche est bien de montrer comment des dispositifs de pouvoir s'articulent directement sur le corps, sur des corps, des fonctions, des processus physiologiques, des sensations, des plaisirs $»^{56}$.

C'est cependant dans les premières œuvres de Foucault, comme Naissance de la clinique, qui ne sont certes pas les plus fréquentées par les «body criticists », que s'impose la présence massive, matérielle, du corps. Dans Naissance de la clinique le corps est loin de n'être qu'une construction ou un langage. Il existe même sous sa forme la plus répulsive, la moins humanisée, qui est celle du «cadavre » et c'est pourtant de ce cadavre que vient la lumière de la connaissance du vivant. Le corps devient en effet accessible au « regard médical moderne » grâce à l'émergence de l'anatomie pathologique et Foucault reprend avec enthousiasme le mot d'ordre qui fut celui de Bichat : «ouvrez quelques cadavres et vous verrez aussitôt disparaître l'obscurité que la seule observation n'avait pu dissiper $\aleph^{57}$. C'est l'ouverture des cadavres, dont la décomposition laisse à voir l'individualisation des

52 Comte, A., « Lettre à Audiffrent du 22 juin 1855 », Correspondance générale et confessions, t. VIII., 18551857, Paris, Editions de l'EHESS-Vrin, 1990 p. 64.

53 Comte, A., Système de politique positive, t. IV, Paris, Carilian-Goeury et Victor Dalmont,1854, p. 36.

54 Cf. Hagner, M., Des cerveaux de génie. Une histoire de la recherche sur les cerveaux d'élite, Paris, Editions de la Maison des sciences de l'homme, 2008.

55 Foucault, M., La volonté de savoir, Paris, Gallimard, 1976, p. 200.

56 Ibíd., p. 200.

57 Bichat, X., Anatomie générale, avant-propos, citado en : Foucault, M., Naissance de la clinique. Une archéologie du regard médical (1963), Paris, P.U.F., 1975, p. 149. 
tissus et leur comparaison possible, qui fournit à l'analyse de type idéologique un modèle «indépassable», plus complet que celui qui est offert par la chimie ou les mathématiques : « la décomposition idéologique ne peut être que la répétition dans la conscience du médecin de celle qui sévit dans le corps du malade ${ }^{58}$. Il commente cette découverte de manière poétique : " la nuit vivante se dissipe à la clarté de la mort ${ }^{59}$. Plus précisément c'est le corps énigmatique qui s'ouvre à la connaissance grâce à la mort : « la mort, au contraire, ouvre à la lumière du jour le noir coffre des corps $»^{60}$.

Grâce à la théorie tissulaire de Bichat le «regard médical» peut s'arrêter et insister sur le corps du malade, ses organes mais surtout ses «membranes », c'est-à-dire ses tissus. Quand Foucault définit ce regard comme un « regard loquace » c'est « parce que ce regard a cette particularité d'entendre un langage au moment où il perçoit un spectacle» ${ }^{61}$. Foucault veut dire par là que la « grammaire des tissus » élaborée par Bichat impose une nouvelle spatialisation du corps. Le Traité des membranes invente une «lecture diagonale du corps, qui se fait selon des nappes de ressemblance anatomique, qui traversent les organes, les enveloppent, les divisent, les composent et les décomposent, les analysent et en même temps les lient $»^{62}$. Mais il ne s'agit en aucun cas avec cette notion de regard loquace d'affirmer que les corps ne sont que langage, Foucault souligne au contraire que le modèle condillacien de « décomposition » et de « composition » des tissus doit permettre de retrouver finalement un réel dont « l'armature est elle-même dessinée d'après le modèle du langage ${ }^{63}$.

Au-delà de Bichat c'est l'œuvre de Broussais qui permettra au corps d'exister véritablement pour l'Ecole médicale de Paris sans un espace idéal des maladies en arrière-plan. Broussais, que Foucault réévalue complétement, a fixé pour son époque le «dernier élément de la manière de voir », il achevé la constitution du « regard médical moderne ${ }^{64}$. Alors que Bichat ne se prononçait pas sur l'être même de la maladie, Broussais, dans son combat continuel contre l' « ontologie» médicale, réduit radicalement l'espace causal des maladies à l'espace local des « organes souffrants ». Le corps, rien que le corps, il n'existe pas d' « entités morbides » générales, pas même de maladies à proprement parler, seulement des « cris des organes souffrants ». On connaît l'impératif que Broussais assigne au médecin dans un passage fameux de l'Examen de la doctrine médicale généralement adoptée de 1816 : « formez un tableau aussi vrai qu'animé du malheureux livré aux angoisses de la douleur ; débrouillez moi, par une savante analyse, les cris souvent confus des organes souffrants ; faites moi connaître leurs influences réciproques ; dirigez habilement mon attention vers le douloureux mobile du désordre universel qui frappe mes sens afin que j'aille y porter avec sécurité le baume consolateur qui doit terminer cette scène déchirante, alors j'avouerai que vous êtes un homme de génie ${ }^{65}$.

58 Foucault, M. Naissance de la Clinique. Une archéologie du regard médical, p. 131.

59 Ibíd., p. 149.

60 Ibíd., p. 170.

61 Ibíd., p. 108.

62 Ibíd..., p. 130.

63 Ibíd.., p. 96.

64 Ibíd.., p. 197.

65 Broussais, F. J. V., Examen de la doctrine médicale généralement adoptée, Paris, Méquignon-Marvis, 1816, p. 179-180. 
Le symptôme devient signe, dans la mesure où il n'y a plus d'essence de la maladie derrière le symptôme, qu'il conviendrait d'interpréter : la maladie est donnée là dans un signe sans arrière-plan : "désormais le signifiant (signe et symptôme) sera entièrement transparent pour le signifié qui apparaît, sans occultation ni résidu, en sa réalité même» ${ }^{66}$. Foucault résume cette nouvelle « manière de voir » de l'Ecole de Paris par « le changement infime et décisif qui a substitué la question : «Qu'avez-vous?» par quoi s'inaugurait au XVIIIe siècle le dialogue du médecin et du malade avec sa grammaire et son style propres, cette autre où nous reconnaissons le jeu de la clinique et le principe de tout son discours : «Où avez-vous mal ?» ${ }^{67}$.

C'est dans ce contexte qu'apparaît l'autre image par laquelle Foucault désigne le corps, celle de la «pierre noire ». La nouvelle clinique s'aide de ce que Foucault appelle des « mythes épistémologiques » (structure alphabétique de la maladie, réduction nominaliste de l'être de la maladie, modèle chimique de l'analyse, regard sensible et concret du médecin pensé sous la notion de «coup d'œil » médical) pour « pénétrer dans de nouveaux espaces, où la visibilité s'épaissit, se trouble, où le regard se heurte à des masse obscures, à d'impénétrables volumes, à la pierre noire du corps $»^{68}$. Quelques pages plus loin il retrouve les mêmes mots : «l'âge de Bichat » est celui où « l'expérience clinique s'arme pour explorer un nouvel espace, l'espace tangible du corps, qui est en même temps cette masse opaque où se cachent des secrets, d'invisibles lésions et le mystère même des origines ${ }^{69}$.

Parler de pierre noire, c'est donc insister sur une présence insistante, pesante, énigmatique qui est celle du corps et que le nouveau regard médical tente de dévoiler. Pierre noire, masse opaque, mystère des origines, le moins que l'on puisse dire est que pour Foucault le corps est loin d'être devenu transparent, plastique et malléable à volonté. Même si la clinique s'efforce de dévoiler ses mystères, il semble qu'il ait gardé toute son impénétrabilité, voire même sa sacralité.

\section{Le corps comme « donné » et comme « produit »}

C'est d'ailleurs ce que l'on pourrait déplorer dans cette approche du corps par Foucault : une situation de « ou bien / ou bien ». Ou bien un corps-bulle de savon, léger et transparent, ou bien un corps-pierre noire, massif et opaque. Une telle approche nous semble trop radicale dans un sens ou dans l'autre et on pourrait alors être tenté de trouver une vision plus équilibrée du corps, notamment dans l'œuvre de Georges Canguilhem. Georges Canguilhem, bien que médecin, ou parce qu'il est médecin, a justement fort peu écrit sur le sujet du corps alors que toute son œuvre est consacrée à la question de la norme, à celle de la santé et de la maladie. Il n'a que rarement écrit sur le corps, qui n'est pas un problème médical en tant que tel, sauf dans son article sur La santé. Concept vulgaire et question philosophique, qui date de 1988. Ce qui fait l'originalité du « corps vivant» par rapport à la machine, c'est qu'il est « cet existant singulier dont la santé exprime la qualité des pouvoirs qui le constituent en tant qu'il doit vivre sous imposition de tâches, donc en relation d'exposition à un

66 Ibíd., p. 90.

67 Ibíd., p. XIV.

68 Ibíd.,p. 118.

69 Ibíd., p. 123. 
environnement dont il n'a pas d'abord le choix. Le corps humain vivant est l'ensemble des pouvoirs d'un existant ayant capacité d'évaluer et de se représenter à lui-même ces pouvoirs, leur exercice et leurs limites $\gg^{70}$. Mais ce corps n'est pas seulement l'ensemble de ces « pouvoirs », il n'est pas que construit : «ce corps est, à la fois, un donné et un produit. Sa santé est, à la fois, un état et un ordre. Le corps est un « donné », dans la mesure où il est un génotype, effet à la fois nécessaire et singulier des composants d'un patrimoine génétique. Sous ce rapport la vérité de sa présence au monde n'est pas inconditionnelle. Il advient parfois des erreurs de codage génétique qui, selon les milieu de vie, peuvent ou non déterminer des effets pathologiques » ${ }^{71}$. Mais le corps est aussi un produit « dans la mesure où son activité d'insertion dans un milieu caractéristique, son mode de vie choisi ou imposé, sport ou travail, contribue à façonner son phénotype, c'est-à-dire à modifier sa structure morphologique et partant à singulariser ses capacités $\gg^{72}$. Le corps est un donné, une chose et un produit, une signification.

Le corps n'est pas qu'un artefact mais ce n'est pas non plus qu'une chose. C'est une chose mais qu'il est en notre pouvoir de modifier, dans certaines limites. C'est ce caractère de « chose » du corps qu'a trop souvent oublié la réflexion phénoménologique sur le corps, au travers du thème de la chair. Comme le note le phénoménologue Jocelyn Benoist, collègue et ami de l'Université de Paris 1 : «si la phénoménologie a quelque chose de particulier à dire du corps [...] c'est que le corps n'est pas une chose. Or il y a dans cette idée quelque chose de tout à fait problématique ». En effet « il est tout à fait essentiel au corps (si ce mot doit signifier ce qu'il signifie ordinairement) qu'il puisse toujours aussi être une chose, et même qu'il le soit toujours» ${ }^{73}$. La « Chair », comme l'avaient aussi noté Deleuze et Guattari, est « pour Husserl et beaucoup de ses successeurs » un moyen de « continuer le travail de taupe du transcendant dans l'immanence elle-même ${ }^{74}$.

$* * *$

De ce point de vue il semble que certains artistes aient mieux compris ce double caractère du corps : transformable et déformable dans une certaine mesure mais pas escamotable ou disponible à volonté, matériel mais aussi doté de signification. Je pense bien sûr à Francis Bacon qui a tout à la fois compris que les corps, qui «crient » et sont défigurés, sont de la «viande », de la pure matière, mais qu'ils ne sont pas méprisables pour autant, qu'ils ont même une dimension spirituelle. Cette « viande » a au contraire chez lui une sorte de dimension religieuse : «J'ai toujours été très touché », disait-il dans ses entretiens avec David Sylvester, «par les images relatives aux abattoirs et à la viande, et pour moi elles sont liées étroitement à tout ce qu'est la Crucifixion. C'est sûr, nous sommes de la viande, nous sommes des carcasses en puissance. Si je vais chez un boucher, je trouve toujours

70 Canguilhem, G. « La santé : concept vulgaire et question philosophique », en : Canguilhem, G., Ecrits sur la médecine, Paris, Editions du Seuil, 2002, p. 58-59.

71 Ibíd., p. 59.

72 Ibíd., p. 59.

73 Benoist, J., « Phénoménologie », en : Marzano, M., Dictionnaire du corps, Paris, P.U.F., 2007, p. 710.

74 Deleuze, G., Guattari, F., Qu'est-ce que la philosophie?, Paris, Editions de Minuit, 1991, p. 48. 
surprenant de ne pas être là, à la place de l'animal» ${ }^{75}$. Ce que Deleuze commente alors: «ce que la peinture de Bacon constitue, c'est une zone d'indiscernabilité, d'indécidabilité, entre l'homme et l'animal. [...] Cette zone objective d'indiscernabilité, c'était déjà tout le corps, mais le corps en tant que chair ou viande. [...] Pitié pour la viande ! Il n'y a pas de doute, la viande est l'objet le plus haut de la pitié de Bacon, son seul objet de pitié, sa pitié d'AngloIrlandais. [...] La viande n'est pas une chair morte, elle a gardé toutes les souffrances et pris sur soi toutes les couleurs de la chair vive. Tant de douleur convulsive et de vulnérabilité, mais aussi d'invention charmante, de couleur et d'acrobatie Bacon ne dit pas « pitié pour les bêtes », mais plutôt tout homme qui souffre est de la viande [...]. Le peintre est boucher certes, mais il est dans cette boucherie comme dans une église, avec la viande pour Crucifié $»^{76}$. Une « viande spirituelle », peut-être est-ce en définitive cela, notre corps ...

\section{Bibliografía}

ANDERS, G. (2002): L'obsolescence de l'homme, Paris, Editions de l'Encyclopédies des Nuisances - Editions Ivrea, Paris.

BENOIST, J. (2007): « Phénoménologie », en : Marzano, M., Dictionnaire du corps, Paris, P.U.F.

BORDO, S. (2003): Unbearable Weight: Feminism, Western Culture, and the Body, Berkeley-Los Angeles, University of California Press.

BROUSSAIS, F. J. V. (1816): Examen de la doctrine médicale généralement adoptée, Paris, Méquignon-Marvis.

BUTLER, J. (2005): Trouble dans le genre, Paris, La Découverte.

BUTLER, J. (2009): Des corps qui comptent, Paris, Editions Amsterdam.

BYNUM, C. (1995): «Why All the Fuss about the Body? A Medievalist's Perspective », Critical Inquiry, 22, pp. 1-33.

CANGUILHEM, G. (2002): «La santé : concept vulgaire et question philosophique », en : Canguilhem, G., Ecrits sur la médecine, Paris, Editions du Seuil.

COMTE, A. (1854): Système de politique positive, t. IV, Paris, Carilian-Goeury et Victor Dalmont.

COMTE, A. (1990): «Lettre à Audiffrent du 22 juin 1855 », Correspondance générale et confessions, t. VIII., 1855-1857, Paris, Editions de l'EHESS-Vrin.

COURTINE, J.-J. (2006): «Introduction » en : Corbin, A., Courtine J.-J., Vigarello, G. (eds) : Histoire du corps, t. 3, Les mutations du regard. Le XXe siècle, Paris, Editions du Seuil.

CRONENBERG, D. (2000): Entretiens avec Serge Grunberg, Paris, Les Cahiers du Cinéma.

DELEUZE, G. (1981): Francis Bacon. Logique de la sensation, Paris, Editions de la différence, t. I.

DELEUZE, G., GUATTARI, F. (1991): Qu'est-ce que la philosophie ?, Paris, Editions de Minuit.

EWING, A. (2000): Le siècle du corps, Paris, Editions de La Martinière.

75 Bacon, F. L'art de l'impossible. Entretien avec David Sylvester, citado en : Deleuze, G., Francis Bacon. Logique de la sensation, Paris, Editions de la différence, 1981, t. I, p. 21.

76 Ibíd., p. 19-21. 
FASSIN, E. (2008): «L'empire du genre. L'histoire politique ambiguë d'un outil conceptuel », en : L'Homme, 2008/3 n 187-188, pp. 375-392.

FAUSTO-STERLING, A. (2000): Sexing the Body. Gender Politics and the Construction of Sexuality, New York, Basic Books.

FECHNER, G. (1997): Anatomie comparée des anges, Paris, Editions de l'éclat.

FOUCAULT, M. (1975a): Naissance de la clinique. Une archéologie du regard médical [1963], Paris, P.U.F.

FOUCAULT, M. (1975b): Surveiller et punir. Naissance de la prison, Paris, Gallimard.

FOUCAULT, M. (1976): La volonté de savoir, Paris, Gallimard.

FOUCAULT, M. (1994): «Les rapports de pouvoir passent à l'intérieur des corps » en : Foucault, M., Dits et écrits, t. III, 1976-1979, Paris, Gallimard.

FOUCAULT, M. (2003): Le Pouvoir psychiatrique. Cours au Collège de France, 1973-1974, Paris, Gallimard-Seuil.

FOUCAULT, M. (2009): Le corps utopique suivi de Les hétérotopies, Paris, Lignes.

GIBSON, W. (1985): Neuromancien, Paris, La Découverte.

GOLDIE, T. (2015): The Man Who Invented Gender. Engaging the Ideas of John Money, Vancouver-Toronto, UBC Press.

HACKING, I.: «Résumé du cours 2003-2004 au Collège de France. Le corps et l'âme au début du XXIe siècle », Site du Collège de France : https://www.college-defrance.fr/ media/ianhacking/UPL2883502379452881562_Hacking2003_2004.pd

HACKING, I. (2007): « Our Neo-Cartesian Bodies in Parts », Critical Inquiry, Vol. 34, N. 1 (Autumn 2007), pp. 78-105.

HAGNER, M. (2008): Des cerveaux de génie. Une histoire de la recherche sur les cerveaux d'élite, Paris, Editions de la Maison des sciences de l'homme.

HOUELLEBECQ, M. (2010): La carte et le territoire, Paris, Flammarion.

LOCK, M., FARQHAR, J., ed. (2007): Beyond the Body Proper, Durham-London, Duke University Press.

MAISONNEUVE, J. (1976): « Le corps et le corporéisme aujourd'hui », en : Revue française de sociologie, 1976, 17-4, pp. 551-571.

MINSKY, M. (1999): «How Computer Science Will Change Our Lives », Nara Conference on Artificial Life (1996) en : Hayles, N. K., How We Became Posthuman, Chicago-London, University of Chicago Press, 1999.

MONEY, J., JOBARIS, R., FURTH, G. (1977): « Apotemnophilia: Two Cases of SelfDemand Amputation as a Paraphilia », Journal of Sex Research 13, no. 2 (1977), pp : $115-25$.

SAMMON, P. M. (1981): « David Cronenberg. Article and interview», Cinefantastique, $10: 4$, Spring 1981, 22.

SFORZINI A. (2014): Michel Foucault. Une pensée du corps, Paris, P.U.F.

SHARP, L. (2006): Strange Harvest. Organ Transplants, Denatured Bodies and the Transformed Self, Berkeley-Los Angeles-London, University of California Press.

SZASZ, T. (1979): « Male and Female Created He Them », New York Times, June 10, 1979. 